\title{
Unpredictable hummingbirds: Flight path entropy is constrained by speed
}

2 and wing loading

\author{
Ilias Berberi ${ }^{1}$, Paolo S. Segre ${ }^{2}$, Douglas L. Altshuler ${ }^{3}$, and Roslyn Dakin ${ }^{1}$
}

4

${ }^{1}$ Department of Biology

6 Carleton University

1125 Colonel By Drive

8 Ottawa, Ontario, Canada K1S 5B6

$10 \quad{ }^{2}$ Hopkins Marine Station

Stanford University

12120 Ocean View Blvd.

Pacific Grove, CA, USA 93950

14

${ }^{3}$ Department of Zoology

16 University of British Columbia 4200-6270 University Blvd.

18 Vancouver, BC, Canada V6T 1Z4

20 To whom correspondence should be addressed:

Roslyn Dakin

22 roslyn.dakin@gmail.com

24 Keywords: Animal flight, locomotion, sequence analysis, repeatability, biomechanics, predictability 


\section{ABSTRACT}

Unpredictable movement can provide an advantage when animals avoid predators and other

28 threats. Previous studies have examined how varying environments can elicit unpredictable movement, but the intrinsic causes of complex, unpredictable behavior are not yet known. We

30 addressed this question by analyzing $>200$ hours of flight performed by hummingbirds, a group of aerial specialists noted for their extreme agility and escape performance. We used information

32 theory to calculate unpredictability based on the positional entropy of short flight sequences during 30-min and 2-hour trials. We show that a bird's entropy is repeatable, with stable

34 differences among individuals that are negatively correlated with wing loading: birds with lower wing loading are less predictable. Unpredictability is also positively correlated with a bird's

36 overall acceleration and rotational performance, and yet we find that moment-to-moment changes in acceleration and rotational velocities do not directly influence entropy. This indicates

38 that biomechanical performance must share an underlying basis with a bird's ability to combine maneuvers into unpredictable sequences. Contrary to expectations, hummingbirds achieve their

40 highest entropy at relatively slow speeds, pointing to a fundamental trade-off whereby individuals must choose to be either fast or unpredictable. 


\section{INTRODUCTION}

One of the most effective ways to avoid predators and other threats is to escape by moving in an

44 unpredictable way [1-3]. Previous studies have tested how environmental factors, such as the presence of a predator, can cause individuals to temporarily become less predictable [4-9].

46 Experiments have also shown that unpredictability can be advantageous, both to escaping animals [10] and those in pursuit of prey [11]. However, it is not yet known whether some

48 individuals are inherently more unpredictable than others, as a result of inter-individual differences in the underlying control systems. These inter-individual differences are necessary

50 for selection to act on unpredictable behavior $[3,12]$.

Biomechanical traits, such as morphology and muscle composition, are one potential

52 driver of individual differences in unpredictability [7,13]. Morphology strongly predicts the locomotor capabilities of individuals [14,15] and covaries with evolved behavioral differences

54 across species [9,16-18]. An animal's morphology and muscle composition also determine limb kinematics that influence whole-body maneuvers [19-21]. These maneuvers are combined as

56 sub-units to generate longer sequences of behavior that may vary in higher-order properties such as unpredictability [9] (Fig. 1). The available data suggest two non-exclusive hypotheses for the

58 relationship between morphology, biomechanical traits, and unpredictable behavior. The first hypothesis, which we refer to as the facilitation hypothesis, is that enhanced power-generation may be associated with greater unpredictability $[9,22,23]$. This can occur if high-performance maneuvers directly confer unpredictability, or if there is a shared underlying basis for

62 maneuverability and unpredictability [17]. For example, more maneuverable individuals may have sensory or neuromuscular advantages that also allow them to combine behavioral sub-units

64 in more unpredictable ways. The second hypothesis, which we refer to as the trade-off 
hypothesis, is that power generation may be negatively associated with unpredictability [7]. This

can occur if high-performance maneuvers restrict an animal's ability to vary its subsequent trajectory [24], due to constraints of inertia and/or sensory and neuromuscular processing.

68 Testing these broad hypotheses is challenging, because it requires tracking individuals that are free to perform diverse maneuvers and combine them in different ways. extremely agile in three dimensions, excellent at evading threats, and their survival and mating

72 success often depend on locomotor performance [25]. One of the primary reasons that hummingbirds are so agile is their extremely high wingbeat frequencies (up to $80 \mathrm{~Hz}$ ), which

74 generate rapid forces on both the upstroke and downstroke [26,27]. This allows hummingbirds to perform a wide range of aerial maneuvers that can be combined into complex sequences of

76 behavior during pursuit and escape [28-30] (Fig. 1b, Table 1). Previous studies of flying hummingbirds have shown that the kinematics of the wings and body are influenced by inter-

78 individual variation in body mass, flight muscle mass, and wing loading [31-33]. More recently, these morphological traits have also been mapped to individual differences in the use and 80 performance of different maneuvers defined in Table 1 [28,29,34]. For example, flight muscle capacity is correlated with enhanced performance on multiple translational, rotational, and

82 turning performance metrics. Reduced wing loading is also associated with enhanced performance of aerial maneuvers. Finally, body mass variation within species has been shown to

84 have a detrimental effect on performance. These previous studies provide a foundation for understanding agility in avian flight, making hummingbirds a tractable system for investigating

86 the mechanistic basis of unpredictable movement. 
Here, we test whether variation in unpredictability is related to biomechanical traits and maneuvering performance. We use data from previous studies of hummingbirds where the individual birds were tracked extensively in a large chamber and were free to choose their

90 sequence of flight behaviors. The birds often flew while motivated by the variable presence of the human experimenter or other startling stimuli. This method allowed us to record a range of

92 rapid and ballistic maneuvers, such as those shown in Fig. 1. To evaluate whether unpredictability may be driven by intrinsic among-individual differences, we used positional

94 entropy to quantify unpredictability [8-10] and tested whether it was repeatable amongindividuals. Next, we used a comparative study of 207 individuals from 25 species to evaluate

96 whether unpredictability differs among species and whether it is related to inter-individual differences in morphology and maneuvering performance. According to the facilitation

98 hypothesis, we expected more unpredictable individuals to use higher-performance translations, rotations, and complex turns, whereas we had the opposite expectation under the trade-off hypothesis. Under the facilitation hypothesis, we also expected a bird's burst muscle capacity to be positively associated with unpredictable behavior, and its body mass and wing loading to be

102 negatively associated with unpredictability. Additionally, we examined wing aspect ratio, a trait that is associated with energetic efficiency and with the use of certain turns in hummingbirds 104 [29,35]. If unpredictability requires energetic efficiency, then we expected individuals with higher aspect ratio wings to be more unpredictable. Next, to evaluate whether the use and 106 performance of specific maneuvers directly influences unpredictability, we used a withinindividual analysis. This analysis tested whether moment-to-moment changes in a bird's

108 predictability were associated with specific maneuvers. To provide context for these results, we also used a simulation to test how the speed of behavior theoretically influences unpredictability. 


\section{MATERIAL AND METHODS}

\section{2 (a) Flight trials and tracking system}

We used tracking data on wild-caught hummingbirds that were recorded in two previous studies:

114 (1) a repeatability study in which the same 20 individuals were assayed in multiple flight trials [28], and (2) a comparative study that assayed 207 individuals from 25 species [29]. Each study captured individuals at multiple sites ( 3 sites for repeatability and 4 for comparative, described in $[28,29])$. To assay flight behavior, the birds were tracked during flight trials in a large $3 \mathrm{~m} \times 1.5$

$\mathrm{m} \times 1.5 \mathrm{~m}$ chamber where they were motivated by a variety of environmental stimuli, including the threat of the human experimenter. In 32 of the trials in the repeatability study, a competing

120 bird was also present. Flight trials lasted for 2 hours in the repeatability study, and 30 min in the comparative study. Flight in the chamber was recorded with four or five synchronized cameras

122 (GE680, Allied Vision Technologies, Stadtroda, Germany) filming at 200 fps. An automated tracking system was used to determine the body position and orientation of the birds in each

124 frame [36]. The output of the tracking system was smoothed using a forward-reverse Kalman filter and ground-truthed by re-projecting the smoothed data onto the videos, to verify that it 126 matched the movements of the animals $[28,34,36]$.

Some of the earliest work conceptualizing unpredictability focused on phenomenological descriptions [3]. Modern computational approaches provide a standardized way to quantify unpredictability using information theory, allowing individuals and species to be compared [8- 
positional entropy, defined as the ability to predict an animal's future position given its past

134 trajectory [37]. Large values of positional entropy correspond to highly unpredictable movement and are indicative of multiple dynamic changes in speed and heading. Positional entropy is

136 calculated based on a series of spatial coordinates in one, two, or three dimensions (see [37] for equations). Mathematically, the computation ignores the scale of the spatial units, such that a

138 sequence of behavior with a given spatial configuration (like a perfect circle with constant velocity) would have the same entropy, regardless of whether it has a $2 \mathrm{~cm}$ or $2 \mathrm{~m}$ radius.

140 However, positional entropy is highly influenced by the temporal duration of the sequence: as the duration gets smaller, the positional entropy asymptotically approaches 0 . Therefore, when

142 using this metric for comparison, it is important to fix the temporal scale to a single duration that can be applied to all individuals.

Because positional entropy is a sequence-based metric, it is challenging to define the start and end points. In our hummingbird free-flight trials, the natural transitions between flight

146 modes and maneuvers were highly variable. We therefore quantified positional entropy using a sliding window approach [38]. We chose a 2-s duration for each window (Fig. 1d), because this

148 duration was long enough to contain multiple maneuvers defined in Table 1 (average $4 \pm 2$ SD), while also occurring many times within each trial. We identified each window at $50 \mathrm{~ms}$ intervals 150 after the start of the trial. Because these sequences are overlapping and consecutive, they are not independent. All three-dimensional positional entropy calculations and further statistical analyses

152 were performed in $\mathrm{R}$ (version 3.6.2) [39].

An important consideration when calculating entropy is that even when the animal is

154 stopped, subtle movements of its body parts or unsmoothed tracking error can produce very high entropy values, because the calculation is normalized on a relative spatial scale. In any study of 
156 whole-body movement, it is therefore important to limit the analysis of positional entropy to sequences when the spatial changes are driven by whole body movement (as opposed to body parts). We used a threshold criterion whereby entropy was only calculated for sequences when the hummingbird's total velocity was maintained above $0.1 \mathrm{~m} / \mathrm{s}$ throughout all frames, to limit our analysis to forward flight.

To determine if unpredictability differs among individuals, we used data from 20 Anna's

164 hummingbirds (Calypte anna) in the repeatability study. All individuals were assayed once on their own, and 1-2 times in the presence of a competitor, as part of a previous experiment to test

166 whether competitor presence affected maneuvering performance [28]. For the present study, we calculated a single trial-average entropy for each bird in a given trial ( $\mathrm{n}=52$ trials). We used a

168 mixed-effects model in the "Ime4" package [40] with bird ID as the random effect to compute the repeatability of trial-average entropy [41]. The model controlled for site (3 levels) and

170 competitor presence (present vs. absent) as the fixed effects. To determine if species differ in unpredictability, we also computed repeatability (at the species level) of trial-average entropy in

172 the comparative study, controlling for site (4 levels; $\mathrm{n}=207$ individuals from 25 species) $[28,29]$. We determined the $95 \%$ confidence intervals of repeatability estimates by parametric

174 bootstrapping with 10,000 iterations using the "bootMer" function in lme4.

To determine how a bird's maneuverability was related to its unpredictability, we analyzed trial178 average entropy in relation to 12 maneuvering performance metrics that were previously shown 
to be repeatable [28] and correlated with each other [29]. A brief description of each metric is provided in Table 1 [28,29]. Although the maneuvers in Table 1 are not a complete list of what hummingbirds can perform, they represent a simple set of behavioral units that are shared by all individuals studied. We first evaluated the association between an individual's trial-average entropy and each performance metric by fitting a separate phylogenetic regression model for each metric, with entropy as the response variable and the performance metric as the predictor. Models were run in the Bayesian "MCMCglmm" package [42] using the most recent multilocus phylogeny from [43], and we included field site as a fixed effect to account for potential site differences.

Next, we evaluated which morphological and muscle traits best explain an individual's overall unpredictability. Measures of body mass, wing loading, and aspect ratio were taken from the same individuals in the maneuvering trials [29]. We obtained estimates of species-average muscle capacity based on previous studies that used a transient load-lifting assay [29,32,44]. This analysis was also run as a Bayesian phylogenetic regression in $\mathrm{MCMCglmm}$, with the following predictors: individual body mass, wing-loading, and aspect ratio, species muscle capacity, and 194 field site.

All MCMCglmm models were run with uninformative priors for Gaussian data. Each 196 chain was run for 500,000 iterations after a burn-in period of 3,000 and the posterior results were thinned to include every $500^{\text {th }}$ sample. We verified that autocorrelations in the posterior samples were $<0.1$ and confirmed that models had converged by checking the Gelman-Rubin statistic for two independently seeded chains. We report standardized effect sizes as the posterior mean \pm $95 \%$ credible intervals. 


\section{(e) Within-individual analysis and simulated effect of speed}

204 Using data from the comparative study, we tested whether particular maneuvers could explain moment-to-moment changes in entropy. We computed a series of additional metrics for each $2 \mathrm{~s}$

206 sliding window: (i) travel velocity, as the sum of the Euclidean distances travelled between each frame, divided by the time $(2 \mathrm{~s})$, (ii-v) the total number of horizontal translational maneuvers,

208 rotational maneuvers, pitch-roll turns, and arcing turns performed in the time window, and (vivii) the average performance on any translational maneuvers $\left(\mathrm{m} / \mathrm{s}^{2}\right)$ and any rotational maneuvers

210 (rev/s) performed in that window. For (vi), AccHor ${ }_{\max }$ and DecHor $_{\max }$ were combined, and

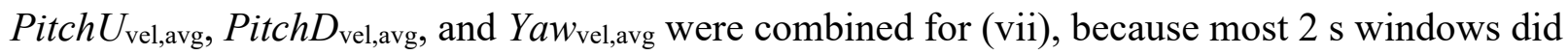
212 not include a maneuver of each type (Table S1).

We fit a linear mixed-effects model in the "nlme" package [45] to analyze entropy for

214 each $2 \mathrm{~s}$ window as a function of the predictors (i-vii) above. The model included nested random effects of species, individual ID, and flight bout to account for non-independence at these three

216 levels. A flight bout included any sequential 2-s windows; after a gap $>50 \mathrm{~ms}$, a new bout was assigned. To account for strong temporal autocorrelation, the model included the corAR1

218 autocorrelation structure [46]. The sample size for this analysis was limited to windows that had at least one translation and one rotation $(\mathrm{n}=251,545$ windows for 205 individuals $)$. We also

220 checked models that dropped average translational and/or rotational performance to verify that the conclusions for the other predictors were unchanged when analyzing the full dataset. assumed that individuals in the 20 solo trials of the repeatability study could perform the same 
faster), we removed every other recorded frame throughout each trial. This method retained the same spatial geometry of the flight paths, but performed it in half the time (see Fig. S1 for an example). To simulate a decrease in speed (2x slower), we added a new frame between each

228 original frame and interpolated the bird's coordinate values. This treatment also retained the same spatial geometry, but performed it in twice as much time (Fig. S1). We recalculated trialaverage entropy based on sliding $2 \mathrm{~s}$ windows in the newly simulated timeframes. To analyze the simulation outcome, we fit a linear mixed effects model in the "lme4" package, with trialaverage entropy as the response variable, speed condition (original, slower, or faster) as the fixed effect, and individual identity as a random effect.

\section{RESULTS}

236 Repeatability and species differences

Some hummingbirds are consistently more unpredictable (higher entropy) than others, as

238 indicated by moderate and significant repeatability (Fig. $2 \mathrm{a} ; \mathrm{R}=51 \%, 95 \% \mathrm{CI}=20-77 \%, \mathrm{p}=$ 0.002). We did not detect a significant effect of competitor presence on entropy (Table S2). Our 240 comparative analysis also indicated that species differ in unpredictability, with inter-species differences accounting for $24 \%$ of the total variance in entropy (Fig. $2 \mathrm{~b}, 95 \% \mathrm{CI}=6-42 \%, \mathrm{p}=$ $2420.002)$

\section{Maneuvers, morphology, and entropy}

The most unpredictable birds used lower average peak velocities $\left(V e l_{\max }\right)$, but higher average

246 peak accelerations and decelerations (AccHor ${ }_{\max }$, DecHor $_{\max }$; Fig. 3a, Table S3). These

contrasting effects were surprising, because individual variation in $V e l_{\max }$ is positively correlated 


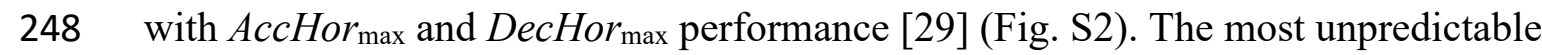
hummingbirds also used faster rotational maneuvers (pitch-up, pitch-down and yaw; Fig. 3a, Fig.

250 S3). Most of the features of the arcing and pitch-roll turns were not strongly related to entropy (Table S3). The exceptions were $P R T_{\text {time }}$ and $P R T \%$ : the most unpredictable hummingbirds

252 tended to perform slightly slower pitch-roll turns and they also used pitch-roll turns in their flight trials more often (Fig. 3a). the most unpredictable hummingbirds had lower wing-loading than others (Fig. 3b, Table S4).

256 Although there was a weak positive relationship between entropy and species muscle capacity, the posterior credible interval included 0 . Body mass and aspect ratio did not explain variation in 258 entropy.

All else being equal, if an animal is able to perform the same sequence of behavior twice as fast,

262 its entropy is expected to increase, whereas entropy is expected to decrease for an animal moving more slowly (Fig. 4a; Table S5). Examining variation in the actual hummingbird trajectories, the

264 best predictor of moment-to-moment changes in entropy was travel velocity. However, contrary to the simulation result described above, hummingbirds were more unpredictable when moving

266 slower (Fig. 4b, Table S6). Entropy within a given flight sequence was also positively associated with translational performance, but not rotational performance (Fig. S4). The lack of rotational effect was surprising, because both translational and rotational performance were strongly associated with inter-individual differences in entropy (Fig. 3a and S4). Furthermore, the 
individual level than it was within individuals (3-to-4 fold difference in effect size; Fig. S5).

272 High entropy sequences also had more rotations and complex turns, but fewer translations (Table S6). However, while these effects were statistically significant, the performance and use of

274 specific maneuvers could only explain $0.4-1.7 \%$ of the variation in unpredictability withinindividuals (Table S6). In contrast, the constraint of travel velocity explained much more of the 276 variation in entropy $\left(\mathrm{R}^{2}\right.$ near $\left.9 \%\right)$.

\section{DISCUSSION}

Our studies of hummingbirds reveal that some individuals and some species are consistently

280 more unpredictable than others (Fig. 2). This raises possibility that unpredictability may be heritable $[12,47]$ and correlated with other intrinsic traits. In our analysis of trial-average

282 entropy, we found strong associations between an individual's unpredictability and biomechanical performance: the most unpredictable hummingbirds used higher-performance

284 accelerations, decelerations, and rotational velocities; they also tended to use pitch-roll turns more often (Fig. 3a). Therefore, hummingbirds with greater capacity for power generation during

286 their maneuvers also move in more unpredictable ways, consistent with the facilitation hypothesis. Given these findings, it was not surprising that a hummingbird's wing-loading was

288 also related to flight path entropy: larger-winged hummingbirds are less predictable (Fig. 3b). Previous research has shown that species with lower wing-loading also perform faster rotations 290 and more sharp turns [29], both of which were also associated with an individual's overall unpredictability in our analysis. However, contrary to the facilitation hypothesis, we found that

292 unpredictability was not related to burst muscle capacity, one of the most important traits that determines power generation for flying hummingbirds [28,32,44]. 
Given the strong associations between an individual's overall maneuverability and entropy (Fig. 3), we expected to find that within individuals, hummingbirds would be less

296 predictable when using fast translational and rotational maneuvers. Surprisingly, we found that moment-to-moment changes in rotational performance were virtually unrelated to entropy, and moment-to-moment changes in translational performance explained less than $2 \%$ of the variation in entropy within individuals (Fig. S4 and Table S6). This indicates that high-performance maneuvers make little to no direct contribution to unpredictability on the scale of our assay. Taken together, these findings imply that among-individual differences in unpredictable flight 302 and high-performance maneuvers must share an underlying basis in some other trait(s) [17]. Possible candidates include a bird's sensory capacities, neuromuscular performance, processing 304 speed, body condition, energy budget, risk tolerance, or skill and expertise [48-50]. An important next step is to determine why we see strong inter-individual correlations between 306 performance and unpredictability, and how these behavioral phenotypes and their underlying systems co-evolve $[17,51]$.

The best predictor of moment-to-moment changes in unpredictability was a bird's speed. Contrary to theoretical expectations, hummingbirds were more unpredictable when flying slowly

310 (Fig. 4). This tradeoff was also observed among individuals: the most unpredictable hummingbirds used high accelerations while maintaining relatively low velocities (Fig. S2),

312 pointing to a fundamental tradeoff between speed and entropy. We propose that flying fast restricts an animal's ability to vary its subsequent trajectory, due to constraints of inertia and/or 314 sensory and neuromuscular processing. These results are similar to ones found in fish [7] and mammals [24,52], suggesting that this trade-off may be a fundamental property of behavioral 316 control systems. 
Overall, we found support for both facilitation and trade-off hypotheses, indicating that

318 the relationship between biomechanics and unpredictability is nuanced. In hummingbirds, speed trades-off with unpredictability, and yet individuals differ in traits (such as relative wing size)

320 that allow some individuals to operate at a higher level of maneuvering performance and unpredictability. Given that the maneuvers themselves do not directly contribute to entropy

322 variation in hummingbirds, our results raise new questions about the mechanisms underlying among-individual differences $[17,49,53]$. Based on our results, we propose that maneuvering

324 performance is associated with a broader biomechanical-behavioral phenotype that also determines unpredictability [9]. Testing the basis of the proposed biomechanical-behavioral

326 phenotype will require high-throughput approaches that capture variation across multiple scales, from sub-units of locomotion to whole sequences of behavior [38]. In addition to the

328 biomechanical and behavioral perspectives, unpredictability carries important fitness consequences $[10,24]$. Future research should explore the intrinsic causes of unpredictability and 330 its tradeoffs using predator-prey and pursuit contexts, where each interaction carries a measurable fitness component [13]. 
334 Table 1. Hummingbird flight maneuvers and performance metrics. For detailed descriptions on all maneuvers see [28].

336

\begin{tabular}{|c|c|c|c|}
\hline Category & Maneuver & Metric & Definition (units) \\
\hline \multirow{3}{*}{ Translation } & 3D Acceleration & $V e l_{\max }$ & Max (peak) velocity in xyz (m/s) \\
\hline & $\begin{array}{l}\text { Acceleration in the horizontal } \\
\text { plane }\end{array}$ & AccHor $_{\text {max }}$ & Max (peak) acceleration in $x y\left(m / \mathrm{s}^{2}\right)$ \\
\hline & $\begin{array}{l}\text { Deceleration in the horizontal } \\
\text { plane }\end{array}$ & DecHor $_{\max }$ & Max (peak) deceleration in $\mathrm{xy}\left(\mathrm{m} / \mathrm{s}^{2}\right)$ \\
\hline \multirow{3}{*}{ Rotation } & Pitch-up body rotation & $P i t c h U_{\text {vel,avg }}$ & Average pitch velocity (rev/s) \\
\hline & Pitch-down body rotation & Pitch $D_{\text {vel,avg }}$ & Average pitch velocity (rev/s) \\
\hline & Yaw turn & $Y a w_{\text {vel,avg }}$ & Average yaw velocity (rev/s) \\
\hline \multirow{6}{*}{$\begin{array}{l}\text { Complex } \\
\text { turns }\end{array}$} & $\begin{array}{l}\text { Sharp, pitch-roll turn in the } \\
\text { horizontal plane }\end{array}$ & $A r c_{\mathrm{rad}}$ & Turn radius $(\mathrm{m})$ \\
\hline & & $A r C_{\mathrm{vel}, \mathrm{avg}}$ & Average velocity in $\mathrm{xy}(\mathrm{m} / \mathrm{s})$ \\
\hline & & $A r c_{\text {cent,max }}$ & Max (peak) centripetal acceleration $\left(\mathrm{m} / \mathrm{s}^{2}\right)$ \\
\hline & $\begin{array}{l}\text { Smooth, arcing turn in the } \\
\text { horizontal plane }\end{array}$ & $P R T_{\text {deg }}$ & Degrees turned $\left(^{\circ}\right)$ \\
\hline & & $P R T_{\text {time }}$ & Duration of turn (s) \\
\hline & $\begin{array}{l}\text { Choice of pitch-roll and arcing } \\
\text { turns }\end{array}$ & PRT\% & $\begin{array}{l}\text { pitch-roll turns/(pitch-roll + arcing } \\
\text { turns)* } 100 \%\end{array}$ \\
\hline
\end{tabular}




\section{FIGURE LEGENDS}

342 Figure 1. Hummingbirds combine simple maneuvers into longer flight sequences. (a) Examples of two 4-s sequences of hummingbird flight, sampled at $200 \mathrm{fps}$. The highlighted

344 points 'Start' and ' $t 4$ ' correspond to the $\mathrm{x}$-axis tick marks on the time series below in (b-d). (b) The timing of translations, rotations, and complex turn maneuvers. The black vertical lines

346 denote the ends of each maneuver. (c) Total velocity vs. time. (d) Positional entropy vs. time, defined as the difficulty of predicting a bird's location during forward flight. Each entropy value is calculated from the surrounding $2 \mathrm{~s}$ window. (e) Entropy boxplots for the two illustrated sequences. The sequence on the left is more predictable than the one on the right (average entropy 0.74 vs. 0.90$)$.

352 Figure 2. Individuals differ in unpredictability. (a) Trial-average entropy was repeatable for 20 male Anna's hummingbirds tested in 2-3 trials (each bird = one column, sorted by individual mean). Circular histograms show headings for the most predictable individual (left) and the most unpredictable individual (right), from a sample of sequences at their most common entropies (vertical line $=0^{\circ}$ heading). Headings were more variable for the high-entropy individual. (b) Species differ in unpredictability. Only the 19 species that had $>2$ individuals are shown.

Figure 3. Unpredictable behavior is associated with an individual's maneuvering performance and wing morphology. (a) Slope estimates $\pm 95 \%$ CI for the relationship between a bird's average entropy and maneuvering performance metrics. See Table 1 for definitions.

362 Positive slopes indicate that higher performance is associated with greater unpredictability $(\mathrm{n}=$ 207 individuals from 25 species). All predictors and the response variable were centered and standardized, so that the values plotted here are comparable effect sizes. Inset examples show that entropy is negatively related to $\mathrm{Vel}_{\max }$ but positively related to AccHor $\mathrm{max}_{\text {max }}$ (b) Unpredictable individuals have lower wing loading (i.e., more wing area per unit body mass). The y-axis shows partial residuals for entropy after accounting for other predictors in the analysis. See Tables S3S4 for details.

370 Figure 4. The trade-off between speed and unpredictability. (a) A simulation tested the effect of performing the same behavioral sequence $2 \mathrm{x}$ slower or $2 \mathrm{x}$ faster. The traces above (a) show position (y-axis) vs. time (x-axis), with tick-marks denoting time points from the original, to show how time is either compressed or expanded (Fig. S1). The boxplot in (a) shows that in

374 principle, faster flight is expected to be less predictable. (b) However, in reality, hummingbirds are more predictable at higher speeds, owing to performance and/or sensory constraints. The blue color is used to represent the frequency of entropy data points per pixel $(n=732,855$ values from 207 birds; the darkest blue indicates 281 values per pixel; white pixels have 0 values). Grey lines show model predictions for each individual's entropy as a function of speed and controlling for temporal autocorrelation. White dots show the predicted values at a bird's median speed. The average number of entropy values per bird was 3,540. For clarity, this plot only shows individuals with at least 2,000 entropy values. See Table S5. 


\section{ETHICS STATEMENT}

384 All research was approved by the Institutional Animal Care and Use Committee of the University of California, Riverside, the University of British Columbia Animal Care Committee,

386 the Ministerio de Agrigultura of Peru, the Ministerio de Ambiente y Energia of Costa Rica, and the Ministerio del Ambiente of the Pichincha province of Ecuador.

DATA ACCESSIBILITY

390 All data and R scripts are available at: https://figshare.com/s/c1c07cd0dc0b8e9a67b1 The repository will be made public when the final version of the study is published.

\section{AUTHOR CONTRIBUTIONS}

394 All authors designed the study. PSS collected the data. RD and IB analyzed the data and wrote the manuscript. All authors edited the manuscript.

396

COMPETING INTERESTS

398 We have no competing interests.

400 FUNDING

Supported by an NSERC Discovery Grant to RD and Carleton University.

402

\section{ACKNOWLEDGEMENTS}

404 We thank the Los Amigos and La Selva Biological Stations, La Georgina restaurant, Hacienda Guaytara, Andrew D. Straw, and numerous field technicians and colleagues who contributed to 406 references $[28,29,34]$. 
408

4101.

4122.

414

416

418

420

422

424

426

428

430

432

434

436

438

440

442

444

446

448

450

452

\section{REFERENCES}

1. Hedenstrom A, Rosén M. 2001 Predator versus prey: On aerial hunting and escape strategies in birds. Behav. Ecol. 12, 150-156. (doi:10.1093/beheco/12.2.150)

2. Lind J, Cresswell W. 2005 Determining the fitness consequences of antipredation behavior. Behav. Ecol. 16, 945-956. (doi:10.1093/beheco/ari075)

3. Humphries DA, Driver PM. 1970 Protean defence by prey animals. Oecologia 5, 285302. (doi:10.1007/BF00815496)

4. Arnott SA, Neil DM, Ansell AD. 1999 Escape trajectories of the brown shrimp Crangon crangon, and a theoretical consideration of initial escape angles from predators. $J$. Exp.

Biol. 202, 193-209.

5. Domenici P, Booth D, Blagburn JM, Bacon JP. 2008 Cockroaches keep predators

guessing by using preferred escape trajectories. Curr. Biol. 18, 1792-1796. (doi:10.1016/j.cub.2008.09.062)

6. Domenici P, Blagburn JM, Bacon JP. 2011 Animal escapology II: Escape trajectory case studies. J. Exp. Biol. 214, 2474-2494. (doi:10.1242/jeb.053801)

7. Hitchcock AC, Chen T, Connolly E, Darakananda K, Jeong J, Quist A, Robbins A, Ellerby DJ. 2015 Trade-offs between performance and variability in the escape responses of bluegill sunfish (Lepomis macrochirus). Biol. Open 4, 743-751.

(doi:10.1242/bio.201511577)

8. Herbert-Read JE, Ward AJW, Sumpter DJT, Mann RP. 2017 Escape path complexity and its context dependency in Pacific blue-eyes (Pseudomugil signifer). J. Exp. Biol. 220, 2076-2081. (doi:10.1242/jeb.154534)

9. Moore TY, Cooper KL, Biewener AA, Vasudevan R. 2017 Unpredictability of escape trajectory explains predator evasion ability and microhabitat preference of desert rodents. Nat. Commun. 8, 1-9. (doi:10.1038/s41467-017-00373-2)

10. Richardson G, Dickinson P, Burman OHP, Pike TW. 2018 Unpredictable movement as an anti-predator strategy. Proc. R. Soc. B Biol. Sci. 285. (doi:10.1098/rspb.2018.1112)

11. Bolton AD, Haesemeyer M, Jordi J, Schaechtle U, Saad FA, Mansinghka VK, Tenenbaum JB, Engert F. 2019 Elements of a stochastic 3D prediction engine in larval zebrafish prey capture. Elife 8, 1-24. (doi:10.7554/eLife.51975)

12. Dochtermann NA, Schwab T, Anderson Berdal M, Dalos J, Royauté R. 2019 The heritability of behavior: A meta-analysis. J. Hered. 110, 403-410. (doi:10.1093/jhered/esz023)

13. Moore TY, Biewener AA. 2015 Outrun or outmaneuver: Predator-prey interactions as a model system for integrating biomechanical studies in a broader ecological and evolutionary context. Integr. Comp. Biol. 55, 1188-1197. (doi:10.1093/icb/icv074)

14. Bennett AF, Garland T, Else PL. 1989 Individual correlation of morphology, muscle mechanics, and locomotion in a salamander. Am. J. Physiol. - Regul. Integr. Comp.

Physiol. 256, R1200-R1208. (doi:10.1152/ajpregu.1989.256.6.r1200)

15. Domenici P. 2001 The scaling of locomotor performance in predator-prey encounters: From fish to killer whales. Comp. Biochem. Physiol. - A Mol. Integr. Physiol. 131, 169182. (doi:10.1016/S1095-6433(01)00465-2)

16. Losos JB. 1990 Concordant evolution of locomotor behaviour, display rate and morphology in Anolis lizards. Anim. Behav. 39, 879-890. (doi:10.1016/S00033472(05)80952-2) 
454 17. Kern EMA, Robinson D, Gass E, Godwin J, Langerhans RB. 2016 Correlated evolution of personality, morphology and performance. Anim. Behav. 117, 79-86.

456 (doi:10.1016/j.anbehav.2016.04.007)

18. Tobalske BW. 2016 Evolution of avian flight: Muscles and constraints on performance. Philos. Trans. R. Soc. B Biol. Sci. 371. (doi:10.1098/rstb.2015.0383)

19. Seebacher F, Pollard SR, James RS. 2012 How well do muscle biomechanics predict whole-animal locomotor performance? The role of Ca2+ handling. J. Exp. Biol. 215, 1847-1853. (doi:10.1242/jeb.067918)

462 20. James RS, Navas CA, Herrel A. 2007 How important are skeletal muscle mechanics in setting limits on jumping performance? J. Exp. Biol. 210, 923-933. (doi:10.1242/jeb.02731)

21. Russell AP, Bels V. 2001 Biomechanics and kinematics of limb-based locomotion in lizards: Review, synthesis and prospectus. Comp. Biochem. Physiol. - A Mol. Integr. Physiol. 131, 89-112. (doi:10.1016/S1095-6433(01)00469-X)

468 22. Moore TY, Rivera AM, Biewener AA. 2017 Vertical leaping mechanics of the Lesser Egyptian Jerboa reveal specialization for maneuverability rather than elastic energy storage. Front. Zool. 14, 1-12. (doi:10.1186/s12983-017-0215-z)

23. Langerhans RB. 2009 Morphology, performance, fitness: Functional insight into a postPleistocene radiation of mosquitofish. Biol. Lett. 5, 488-491. (doi:10.1098/rsbl.2009.0179)

474 24. Wilson AM et al. 2018 Biomechanics of predator-prey arms race in lion, zebra, cheetah and impala. Nature 554, 183-188. (doi:10.1038/nature25479)

476 25. Altshuler DL, Dudley R. 2002 The ecological and evolutionary interface of hummingbird flight physiology. J. Exp. Biol. 205, 2325-2336.

478 26. Warrick D, Hedrick T, Fernández MJ, Tobalske B, Biewener A. 2012 Hummingbird flight. Curr. Biol. 22, R472-R477. (doi:10.1016/j.cub.2012.04.057)

27. Altshuler DL, Dudley R. 2003 Kinematics of hovering hummingbird flight along simulated and natural elevational gradients. J. Exp. Biol. 206, 3139-3147. (doi:10.1242/jeb.00540)

28. Segre PS, Dakin R, Zordan VB, Dickinson MH, Straw AD, Altshuler DL. 2015 Burst muscle performance predicts the speed, acceleration, and turning performance of Anna's hummingbirds. Elife 4, 1-23. (doi:10.7554/eLife.11159)

29. Dakin R, Segre PS, Straw AD, Altshuler DL. 2018 Morphology, muscle capacity, skill, and maneuvering ability in hummingbirds. Science. 359, 653-657.

488 30. Clark CJ. 2011 Effects of tail length on an escape maneuver of the Red-billed Streamertail. J. Ornithol. 152, 397-408. (doi:10.1007/s10336-010-0550-8)

490 31. Hedrick TL, Tobalske BW, Ros IG, Warrick DR, Biewener AA. 2012 Morphological and kinematic basis of the hummingbird flight stroke: Scaling of flight muscle transmission ratio. Proc. R. Soc. B Biol. Sci. 279, 1986-1992. (doi:10.1098/rspb.2011.2238)

32. Altshuler DL, Dudley R, Heredia SM, McGuire JA. 2010 Allometry of hummingbird lifting performance. J. Exp. Biol. 213, 725-734. (doi:10.1242/jeb.037002)

33. Stiles FG, Altshuler DL, Dudley R. 2005 Wing morphology and flight behavior of some North American hummingbird species. Auk 122, 872. (doi:10.1642/00048038(2005)122[0872:wmafbo]2.0.co;2)

498 34. Segre PS, Dakin R, Read TJG, Straw AD, Altshuler DL. 2016 Mechanical constraints on flight at high elevation decrease maneuvering performance of hummingbirds. Curr. Biol. 
26, 3368-3374. (doi:10.1016/j.cub.2016.10.028)

35. Kruyt JW, Quicazán-Rubio EM, Van Heijst GF, Altshuler DL, Lentink D. 2014

502 Hummingbird wing efficacy depends on aspect ratio and compares with helicopter rotors. J. R. Soc. Interface 11, 20140585. (doi:10.1098/rsif.2014.0585)

504

36. Straw AD, Branson K, Neumann TR, Dickinson MH. 2011 Multi-camera real-time threedimensional tracking of multiple flying animals. J. R. Soc. Interface 8, 395-409.

506 (doi:10.1098/rsif.2010.0230)

37. Roberts S, Guilford T, Rezek I, Biro D. 2004 Positional entropy during pigeon homing I:

508

510 Application of Bayesian latent state modelling. J. Theor. Biol. 227, 39-50.

(doi:10.1016/j.jtbi.2003.07.002)

512

38. Ligon RA, Diaz CD, Morano JL, Troscianko J, Stevens M, Moskeland A, Laman TG, Scholes E. 2018 Evolution of correlated complexity in the radically different courtship signals of birds-of-paradise. PLoS Biol. 16, 1-24. (doi:10.1371/journal.pbio.2006962)

39. R Core Team. 2019 R: A Language and Environment for Statistical Computing.

514 40. Bates D, Mächler M, Bolker B, Walker S. 2015 Fitting linear mixed-effects models using \{lme4\}. J. Stat. Softw. 67, 1-48. (doi:10.18637/jss.v067.i01)

516 41. Nakagawa S, Schielzeth H. 2010 Repeatability for Gaussian and non-Gaussian data: A practical guide for biologists. Biol. Rev. 85, 935-956. (doi:10.1111/j.1469-

518 185X.2010.00141.x)

42. Hadfield JD. 2010 MCMCglmm: MCMC methods for multi-response GLMMs in R. $J$. Stat. Softw. 33, 1-22. (doi:10.1002/ana.22635)

522

43. McGuire JA, Witt CC, Remsen J V., Corl A, Rabosky DL, Altshuler DL, Dudley R. 2014 Molecular phylogenetics and the diversification of hummingbirds. Curr. Biol. 24, 910916. (doi:10.1016/j.cub.2014.03.016)

524 44. Altshuler DL, Dudley R, McGuire JA. 2004 Resolution of a paradox: Hummingbird flight at high elevation does not come without a cost. Proc. Natl. Acad. Sci. U. S. A. 101, 1773117736. (doi:10.1073/pnas.0405260101)

528

45. Pinheiro J, Bates D, DebRoy S, Sarkar D, R Core Team. 2019 \{nlme\}: Linear and

46. Zuur A, Ieno EN, Walker N, Saveliev AA, Smith GM. 2009 Mixed effects models and extensions in ecology with $R$. New York: Springer.

532

47. Lessells CM, Boag PT. 1987 Unrepeatable repeatabilities: A common mistake. Auk 104,

48. Altshuler DL, Bahlman JW, Dakin R, Gaede AH, Goller B, Lentink D, Segre PS, Skandalis DA. 2015 The biophysics of bird flight: Functional relationships integrate aerodynamics, morphology, kinematics, muscles, and sensors. Can. J. Zool. 93, 961-975. (doi:10.1139/cjz-2015-0103)

49. Careau V, Garland TJ. 2012 Performance, personality, and energetics: Correlation, causation, and mechanism. Physiol. Biochem. Zool. 85, 543-571. (doi:10.1086/666970)

50. Coppens CM, de Boer SF, Koolhaas JM. 2010 Coping styles and behavioural flexibility: Towards underlying mechanisms. Philos. Trans. R. Soc. B Biol. Sci. 365, 4021-4028. (doi:10.1098/rstb.2010.0217)

542 51. Sih A, Bell AM, Ziemba RE. 2004 Behavioral syndromes: An integrative overview. $Q$. Rev. Biol. 79, 241-277.

544 52. Wynn ML, Clemente C, Nasir AFAA, Wilson RS. 2015 Running faster causes disaster: Trade-offs between speed, manoeuvrability and motor control when running around 
bioRxiv preprint doi: https://doi.org/10.1101/2020.08.11.246926; this version posted August 12, 2020. The copyright holder for this preprint (which was not certified by peer review) is the author/funder, who has granted bioRxiv a license to display the preprint in perpetuity. It is made available under aCC-BY-NC-ND 4.0 International license.

corners in northern quolls (Dasyurus hallucatus). J. Exp. Biol. 218, 433-439. (doi:10.1242/jeb.111682)

548 53. Sih A, Mathot KJ, Moirón M, Montiglio PO, Wolf M, Dingemanse NJ. 2015 Animal personality and state-behaviour feedbacks: A review and guide for empiricists. Trends Ecol. Evol. 30, 50-60. (doi:10.1016/j.tree.2014.11.004) 
bioRxiv preprint doi: https://doi.org/10.1101/2020.08.11.246926; this version posted August 12, 2020. The copyright holder for this preprint (which was not certified by peer review) is the author/funder, who has granted bioRxiv a license to display the preprint in perpetuity. It is made available under aCC-BY-NC-ND 4.0 International license.

\section{Figure 1}

(a)
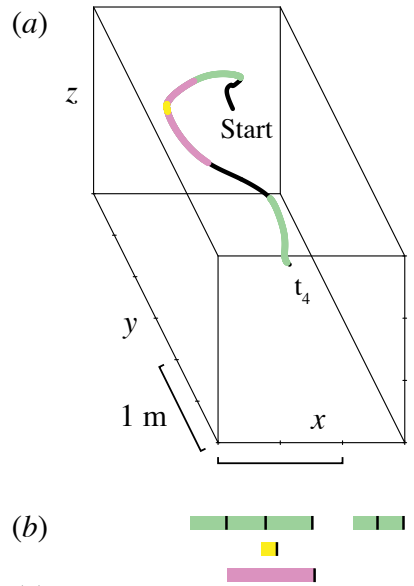

(c)
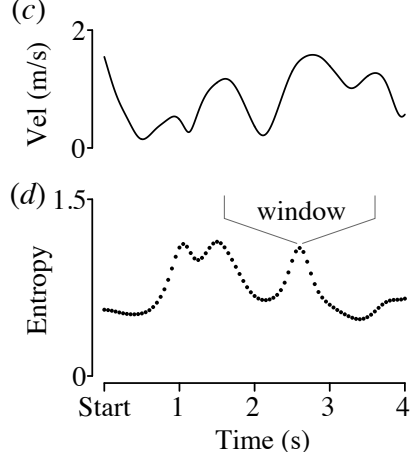
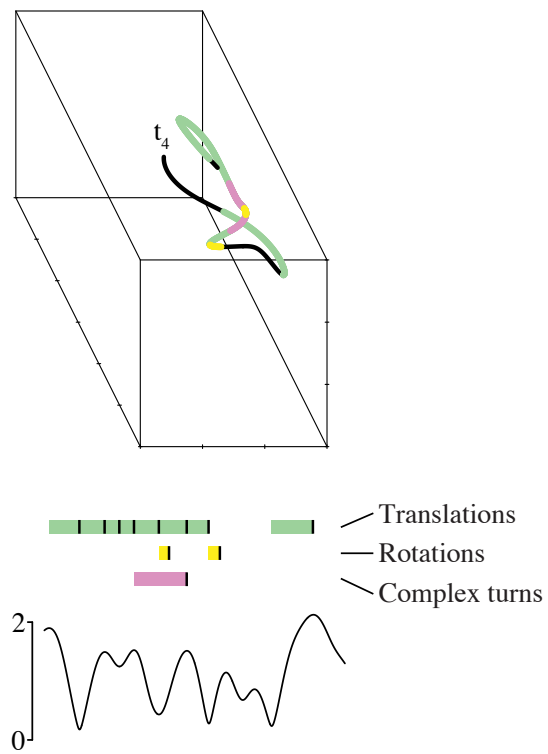

${ }^{1.5}$

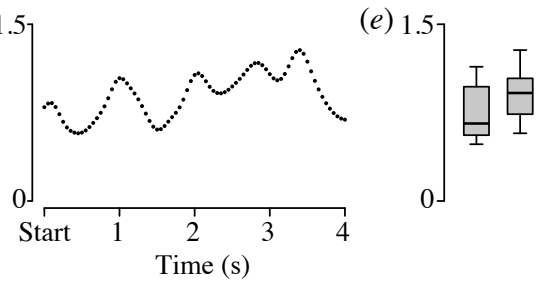




\section{Figure 2}
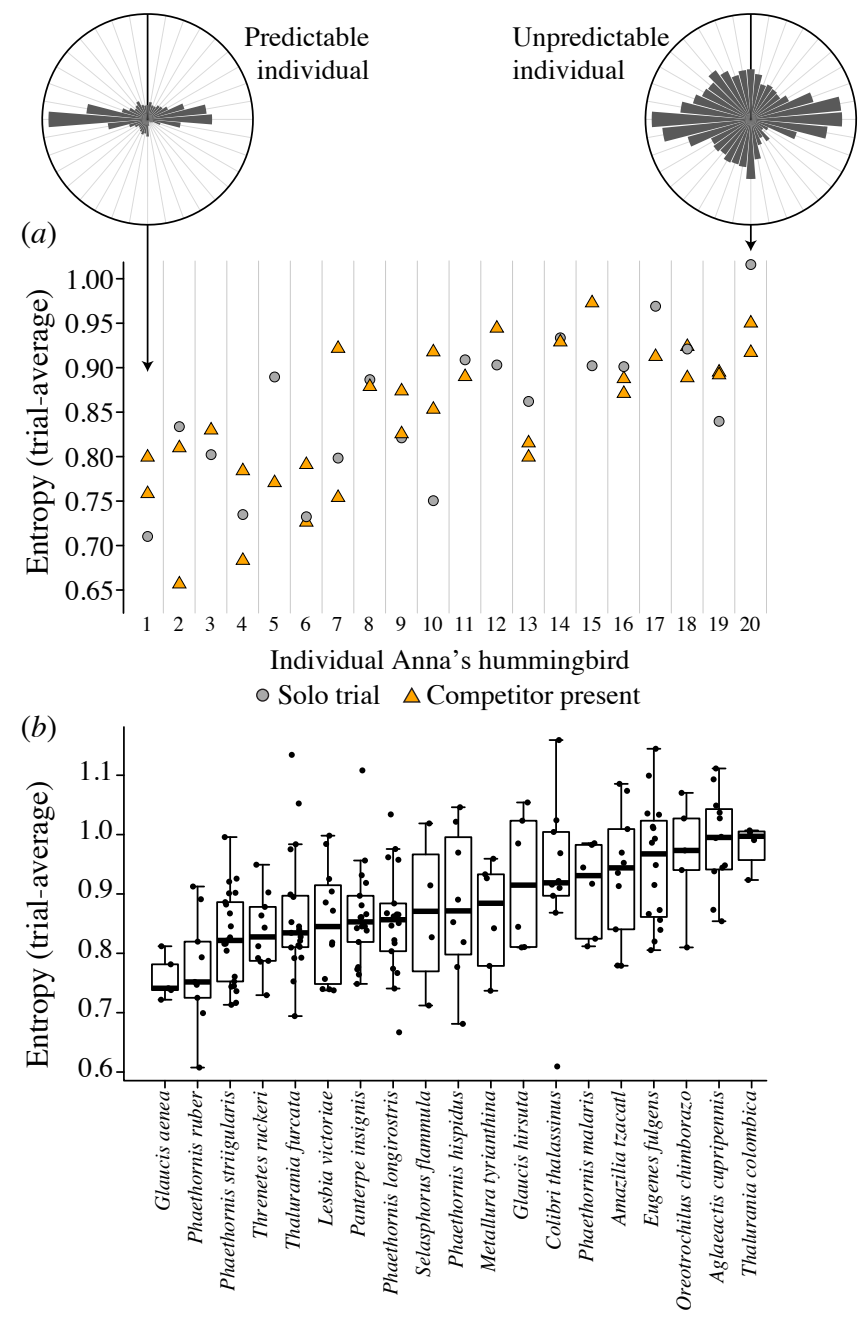


\section{Figure 3}
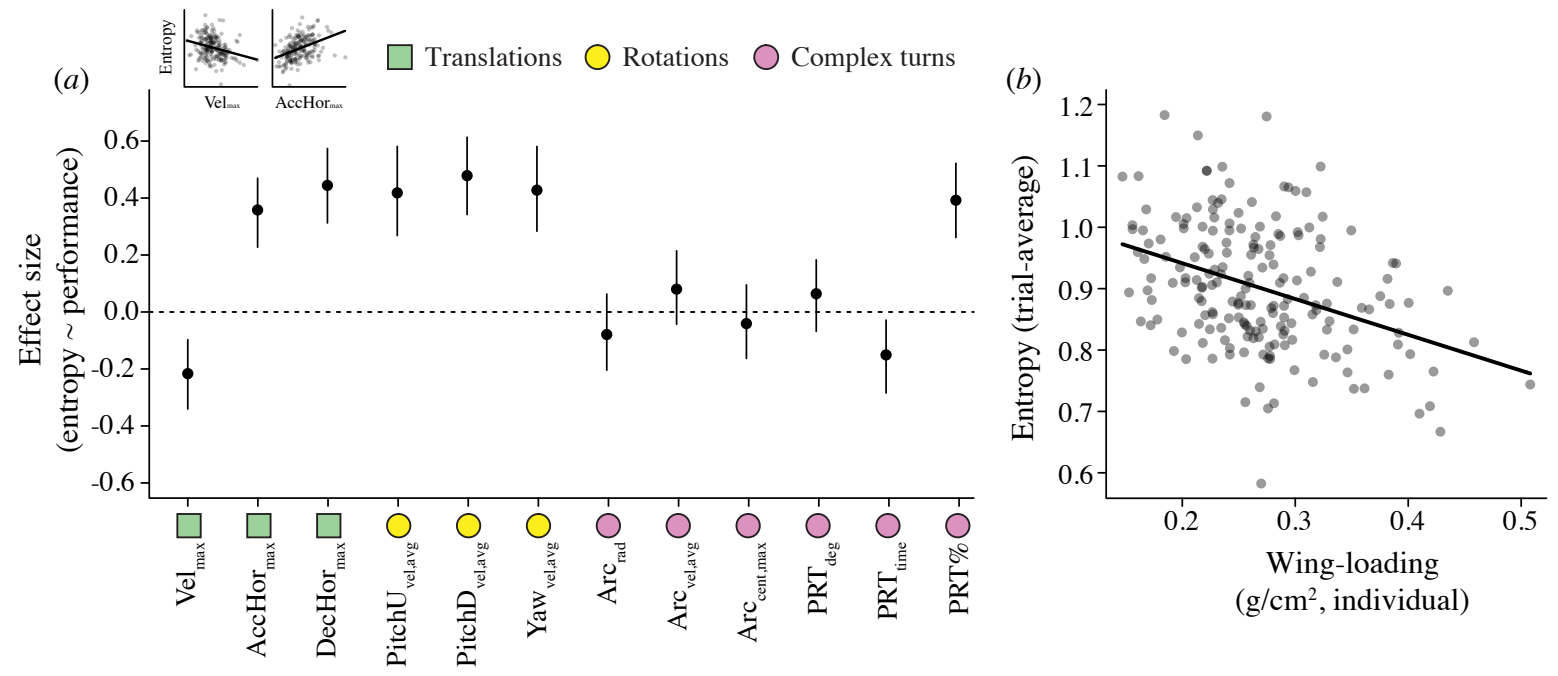
bioRxiv preprint doi: https://doi.org/10.1101/2020.08.11.246926; this version posted August 12, 2020. The copyright holder for this preprint (which was not certified by peer review) is the author/funder, who has granted bioRxiv a license to display the preprint in perpetuity. It is made available under aCC-BY-NC-ND 4.0 International license.

\section{Figure 4}

(a) Simulation

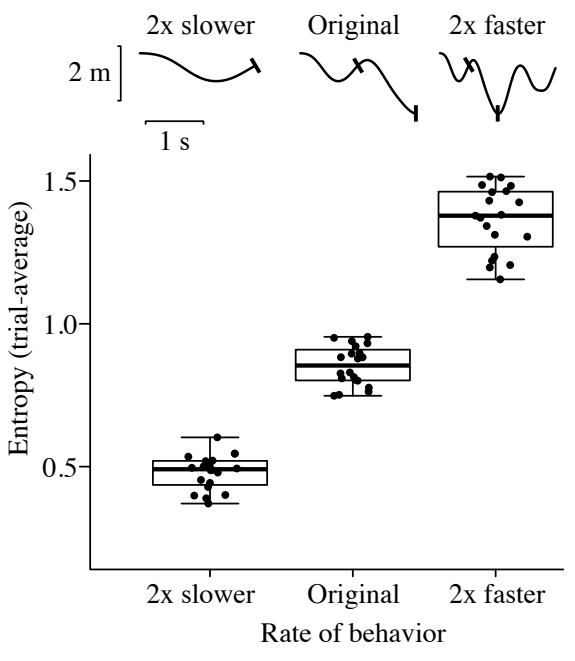

(b) Within-individual analysis

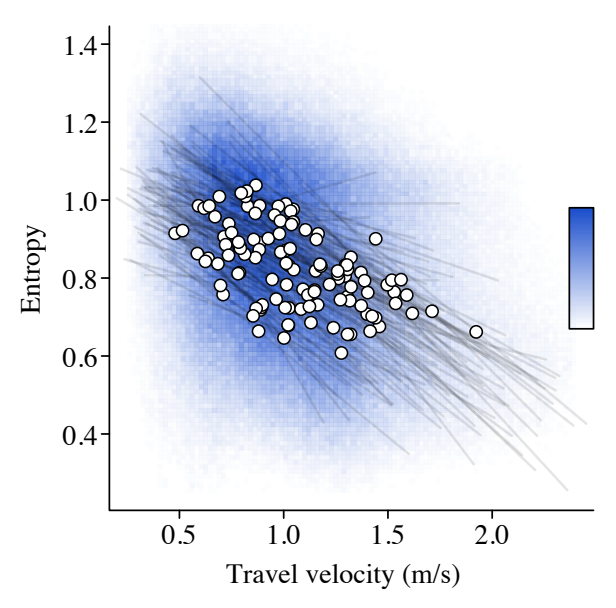

